Running head: Probabilistic cuing of visual search

\title{
Probabilistic cuing of visual search: Neither implicit nor inflexible
}

Tamara Giménez-Fernández ${ }^{1}$, David Luque ${ }^{1,2}$, David R. Shanks $^{3}$, \& Miguel A. Vadillo ${ }^{1}$

${ }^{1}$ Department of Basic Psychology, Autonomous University of Madrid, Spain

${ }^{2}$ Department of Basic Psychology, University of Málaga, Spain

${ }^{3}$ Division of Psychology and Language Sciences, University College London, United Kingdom

\section{Word count: 7,936}

Mailing address:

Miguel A. Vadillo

Departamento de Psicología Básica

Facultad de Psicología

Universidad Autónoma de Madrid

28049 Madrid, Spain

e-mail: miguel.vadillo@uam.es 


\begin{abstract}
In probabilistic cuing of visual search, participants search for a target object that appears more frequently in one region of the display. This task results in a search bias towards the rich quadrant compared with other quadrants. Previous research suggests that this bias is inflexible (difficult to unlearn) and implicit (participants are unaware of the biased distribution of targets). We tested these hypotheses in two preregistered, high-powered experiments $(N s=160$ and 162). In an initial biased stage, participants performed a standard probabilistic cuing task. In a subsequent unbiased stage, the target appeared in all quadrants with equal probability. Awareness questions were included after the biased stage in one group of participants, and after the unbiased stage in a second group. Results showed that participants were aware of the rich area and this effect was larger for the group whose awareness was assessed after the biased stage. In addition, analyses of visual search times indicated that the search bias towards the rich area (formed during the biased stage) was reduced during the unbiased stage. These results cast doubts on the characterization of probabilistic cuing as an implicit and inflexible 'search habit'.
\end{abstract}

Keywords: awareness; false negatives; implicit learning; probabilistic cuing; spatial attention; visual search 
Probabilistic cuing of visual search 3

\section{Public significance statement}

When a target object appears more frequently in one region of a display, visual attention is biased towards that region. This study provides evidence that this phenomenon, called probabilistic cuing, is neither implicit nor inflexible. These results challenge the popular hypothesis that probabilistic cuing constitutes a sort of 'search habit'. Additionally, this study highlights the importance of using adequate sample sizes to prevent false negatives in psychological research. 
The probabilistic cuing task has been used extensively to study how experience shapes the allocation of visual spatial attention (Jiang, 2018). In the standard version of this task (e.g., Jiang, Swallow, Rosenbaum et al., 2013; Jiang et al., 2014), participants search for a visual target among several distractors. The target is more frequently located in one area of the display, although participants are not explicitly instructed about this feature of the task. For example, in half of the trials the target may appear in one specific quadrant of the display (i.e., the rich quadrant), while for the remaining trials it appears evenly distributed across the other three quadrants (i.e., the sparse quadrants). In these experiments, reaction times become faster for trials in which the target appears in the rich quadrant compared to the sparse quadrants.

This result is usually explained as the consequence of a learned visual search bias towards the rich quadrant (e.g., Jiang et al., 2014; Jiang et al., 2018). It has been suggested that this attentional bias is independent of any explicit knowledge that participants may acquire about the uneven distribution of target locations (e.g., Jiang, 2018; Jiang et al., 2018). This hypothesis is partly based on the finding that most participants do not report that the target appeared more frequently in any quadrant and they also seem to perform at chance when asked to identify the rich quadrant (e.g., Geng \& Behrmann, 2002; Jiang et al., 2013, 2014; Jiang, Sha et al., 2015; Jiang, Swallow et al., 2015).

In addition to being implicit or unconscious, it has also been claimed that probabilistic cuing of visual attention is inflexible, in the sense that, once established, the attentional bias persists even when it is no longer advantageous. For instance, when the biased learning stage is followed by an unbiased testing stage in which the target is evenly distributed across the four quadrants, the search bias does not decrease during the unbiased stage (Jiang, Swallow, Rosenbaum et al., 2013). The fact that this type of 
learning is both unconscious and inflexible has led some authors to suggest that it should be understood as an 'attentional habit' (Jiang, 2018) — the attentional equivalent to habitual overt responses acquired through extended practice (e.g., Luque et al., 2019; Wood \& Rünger, 2016).

Contrary to this conclusion, we argue that previous results supporting the unawareness and inflexibility claims are undermined by methodological shortcomings. For instance, the median sample size of previous studies on probabilistic cuing is $N=16$ (for a review, see Vadillo, Linssen et al., 2020). This sample size may be too small to detect a decrease in the magnitude of the probabilistic cuing effect through the unbiased stage, therefore leading to the incorrect conclusion that probabilistic cuing is inflexible. Similarly, these studies might not be sufficiently powered to detect above-chance performance in awareness tests, leading to the incorrect conclusion that participants are unaware. Absence of evidence (failure to reject the null) is not the same as evidence of absence. Consistent with this interpretation, a high-powered meta-analysis of all the studies conducted with this task found that participants' performance in the awareness test is clearly above chance (Cohen's $h=0.35, p<.001$ ), although most studies are underpowered to detect this effect (Vadillo, Linssen et al., 2020; see also Jiang et al., 2018).

Furthermore, the awareness test is usually undertaken after the unbiased stage. When participants are then asked to select the region that contained the target most frequently, it is natural that some of them will fail to select the correct response, either through confusion or through unlearning. After all, the target was equally likely to appear anywhere during the immediately preceding unbiased stage. As Lovibond and Shanks (2002) pointed out, a valid assessment of awareness requires recording conscious content as soon as possible after the target event has taken place (immediacy 
criterion). In the case of the probabilistic cuing task, the inclusion of an unbiased stage may cause awareness about the distribution of targets during the biased stage to be underestimated. Consistent with this, in the meta-analysis by Vadillo, Linssen et al. (2020), experiments that measured awareness immediately after a biased stage $(h=$ $0.52,95 \% \mathrm{CI}[0.32,0.72])$ found greater signs of awareness of the bias than those that measured awareness after the unbiased stage $(h=0.26$, CI $[0.11,0.41])$.

Note, however, that this meta-analytic evidence is only suggestive, at best. The included studies employed heterogeneous methods, coarse awareness measures and small samples. Furthermore, whether studies included an unbiased stage before the awareness test was confounded with other idiosyncrasies of the experimental task, such as the number of regions into which the visual search display was divided or whether the experiment was conducted in a laboratory or a natural setting. Ideally, the impact of an unbiased testing stage on participants' performance in an awareness test should be assessed in a single experiment, rather than in meta-analytic comparisons of heterogeneous experiments. Additionally, we adopt more sensitive measures of awareness than the ones included in the above-mentioned meta-analysis.

Our aim was twofold: First, we explored whether high-powered experiments would show evidence of a reduction in the attentional bias through the unbiased stage (testing the inflexibility hypothesis) and evidence of explicit recognition of the biased spatial distribution (testing the implicitness hypothesis); second, we explored whether administering the unbiased stage just before the awareness test might attenuate the awareness scores. Our preregistered predictions were that, given that our experiment involved a large sample, we would detect a significant decrease in the size of probabilistic cuing through the course of the unbiased testing stage, regardless of whether the unbiased stage was placed before or after the awareness test. Furthermore, 
we expected that participants who completed the awareness test before the unbiased testing stage would show higher levels of awareness than ones who performed it after the unbiased stage. To preview, these predictions were confirmed by the results, posing a serious challenge to the attentional habit theory of probabilistic cuing.

\section{Experiment 1}

As explained above, Experiment 1 was designed to test whether probabilistic cuing of attention declines with extended exposure to an unbiased testing stage. Unlike previous studies exploring this issue, Experiment 1 was conducted with a large sample of participants, which improved considerably the statistical power of this test. In addition, we asked whether the inclusion of an unbiased stage has any effect on participants' awareness scores. Explicit recognition was measured with an alternative test that proved to be more sensitive to awareness in a previous study conducted by our team (Vadillo, Linssen et al., 2020). Specifically, in addition to measuring awareness with a standard measure used in this literature, our awareness test asked participants to rank each quadrant according to the frequency with which it contained the target during the cuing task.

\section{Method}

\section{Participants}

The crucial statistical test for our main hypothesis concerning the recognition test involves a significant interaction in a $2 \times 4$ contingency table assessed through a Chi-square contrast (see Results and Discussion below). Given that the present experiment is only the second one (after Vadillo, Linssen et al., 2020, Experiment 1) to use a ranking test as a measure of awareness and the first one attempting to detect an attenuated interaction with it, it is difficult to conduct any power analysis based on previous evidence. The resources provided by our university permitted us to obtain data 
from approximately 150-160 participants. An a priori power analysis showed that a sample size of 160 participants would grant statistical power of 90 to detect a medium effect size of $w=0.30$, with 3 degrees of freedom and $\alpha=.05$ in a Chi-square test. Thus, one hundred and sixty participants took part in Experiment 1. All participants had normal or corrected to normal vision and completed the experimental task individually in isolated cubicles. All participants were Psychology students from Universidad Autónoma de Madrid (UAM) who provided informed consent and received course credit for their participation. The study was approved by the UAM Ethics Committee (ref. CEI-80-1473).

\section{Stimuli}

On each cuing trial, participants saw a display with $11 \mathrm{~L}$-shaped distractors (which could be rotated $0^{\circ}, 90^{\circ}, 180^{\circ}$, and $270^{\circ}$ ) and one T-shaped target (which could be rotated $90^{\circ}$ or $270^{\circ}$ ). Distractors and targets were positioned in an $11 \times 11$ grid, invisible to participants. This grid was placed inside a $20.7 \mathrm{~cm} \times 20.7 \mathrm{~cm}$ white frame. Each quadrant contained three stimuli (either three distractors or two distractors and the target). All stimuli were presented in white against a black background. The horizontal and vertical lines of all stimuli were $9 \mathrm{~mm}$ in length (Figure 1). The preregistered protocols (methods and analysis plan) are available at https://osf.io/dswkv/.

\section{Procedure and Design}

At the beginning of the experiment, participants were instructed to search for the target (i.e., the only $\mathrm{T}$ in the display) as quickly as possible and press the $\langle\mathrm{z}\rangle$ key if the 


\section{Experiment 1}

Unbiased-first

Awareness-first
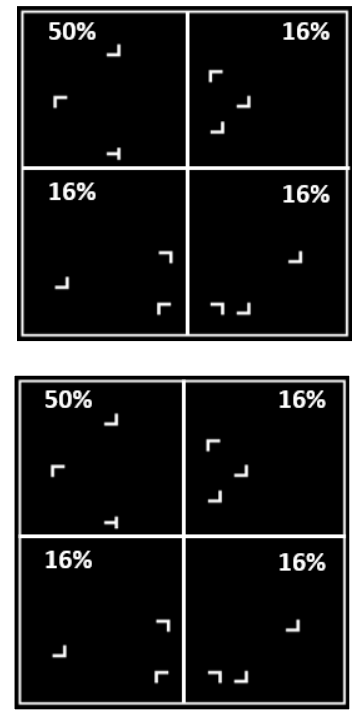

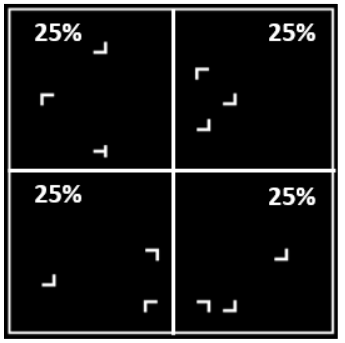

Question 1: Did the T appear with greater

probability in any

quadrant? $(1$ = definitely

not, $\mathbf{6}$ = definitely yes).

Question 2: Rank from

highest to lowest

frequency each of these

quadrants.
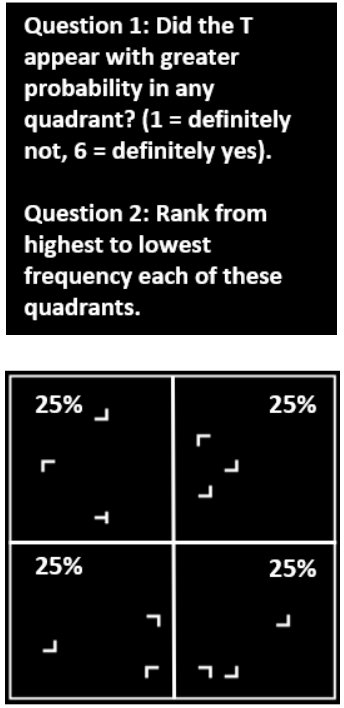

Figure 1. Design of Experiment 1. Dividing lines demarcate the four quadrants and percentages indicate the probability of the target being displayed in each quadrant on a trial. Neither the lines nor the percentages were visible to participants. The Unbiased-first group (top row) completed a biased stage followed by an unbiased stage. Then, they took the awareness test. The Awareness-first group (bottom row) took the awareness test between the biased and unbiased stages.

stem of the T pointed to the left (rotated $90^{\circ}$ ) or $\langle\mathrm{m}>$ if it pointed to the right (rotated $\left.270^{\circ}\right)$. Each trial began with a $1-\sec$ fixation dot presented at the centre of the screen, followed by the search display, which remained visible until the participant's response. After an incorrect response, the message "Error!" was presented on the screen for $3 \mathrm{sec}$. Trials were separated by a 0.5 -sec blank screen. To avoid fatigue, participants could take a rest after trials $95,191,287,383$, and 479 . The left or right orientation of the target was determined randomly in each trial.

The experiment comprised a biased stage followed by an unbiased stage. The awareness test (described below) was presented immediately after the biased stage for half of the participants (Awareness-first group) and immediately after the unbiased stage for the other half of participants (Unbiased-first group). During the biased stage, 
half of the trials contained the target in one quadrant of the display (i.e., the rich quadrant), whereas on the other half of the trials the target was presented in each of the remaining quadrants with equal probability (i.e., the sparse quadrants). The rich quadrant for each participant was randomly assigned at the beginning of the experiment. During the unbiased stage, the target appeared in each quadrant with equal probability (Figure 1). Both the biased and unbiased stages comprised 24 blocks of 12 trials each. Thus, in each block of the biased stage, the target appeared in the rich quadrant on 6 trials (50\% of trials) and in each sparse quadrant on 2 trials (16.7\%). In each block of the unbiased stage, the target appeared three times (25\%) in each quadrant. Participants received no explicit instruction or cue about the location of the target or about the transition from one stage to another.

The awareness test comprised two questions. First, we explained to participants that the search display could be divided into four quadrants. Then, as in most probabilistic cuing experiments, we asked participants: "Did the T appear with greater probability in any quadrant?” [“¿Ha aparecido la T con mayor probabilidad en algún cuadrante?"]. Previous studies of probabilistic cuing have typically asked participants to answer this question with a yes/no response (Jiang et al., 2018). However, we required them to answer it using a rating scale ranging from 1 to 6 , with options labelled “definitely not”, "probably not”, “possibly not”, “possibly yes”, “probably yes”, and “definitely yes" [in Spanish: "seguro que no", “probablemente no", "creo que no", "creo que sí", "probablemente sí" and "seguro que sí"]. We expected that the use of a numerical scale would render the test more sensitive to explicit recognition than the traditional yes/no response format.

Secondly, as in Vadillo, Linssen et al. (2020, Experiment 1), and regardless of their response to the first question, participants were asked to rank each of the four 
quadrants depending on the frequency with which it contained the target during the cuing task. To do this, we informed participants that the target did not appear in all the quadrants with the same frequency. We showed them a square representing the search display divided into four quadrants, together with the following instructions: "We ask you to rank each of these quadrants from highest to lowest frequency" ["Te vamos a pedir que ordenes de mayor frecuencia a menor frecuencia cada uno de estos cuadrantes"]. Each quadrant was labelled with a letter: a, b, c, and d. Participants had to press these letters in descending order, starting with the quadrant they believed had contained the target most often.

\section{Results and Discussion}

Search times. Unless noted otherwise, all the data processing and analyses followed the preregistered protocol—unplanned analyses are explicitly indicated as such. Participants with overall accuracies below 95\% in the visual search task (three in total, two from the Awareness-first group and one from the Unbiased-first group) were removed from the analyses. Thus, 78 and 79 participants were included in the Awareness-first and Unbiased-first groups, respectively. Trials immediately following a rest break (five in total) were discarded, as in previous experiments from our laboratory (Vadillo, Linssen et al., 2020), given that response times (RT) for these trials might be anomalously slow because participants are sometimes not totally ready for the search task. Trials with RTs greater than $10 \mathrm{sec}$ and with incorrect responses were removed from the analyses. Then, for each participant we estimated the mean and standard deviation $(S D)$ of the valid RTs and removed any RT departing by three or more $S D \mathrm{~s}$ from each participant's mean. To further reduce noise in the data, we collapsed data from adjacent blocks into 2-block epochs. Sphericity violations were corrected applying the Greenhouse-Geisser correction when epsilon was lower than 0.75 and the Huynh- 
Feldt correction when epsilon was greater than 0.75 . We report corrected degrees of freedom.

Biased stage. Probabilistic cuing of attention during the biased stage was tested by means of a 2 (Group: Awareness-first vs. Unbiased-first) $\times 2$ (Quadrant: rich vs. sparse $) \times 12($ Epoch $)$ analysis of variance $($ ANOVA) on RTs. Because the groups were treated identically up to and including this stage, we anticipated no main effect or interactions involving the Group factor. The ANOVA yielded a significant main effect of quadrant, $F(1,155)=433.55, p<.001, \eta_{\mathrm{p}}^{2}=.74$, and epoch, $F(6.47,1002.54)=$ $68.15, p<.001, \eta_{\mathrm{p}}^{2}=.31$. All remaining effects were non-significant $(p \mathrm{~s}>.064)^{1}$.

As explained in the preregistered protocol, to assess the possibility that these results were influenced by repetition priming (Walthew \& Gilchrist, 2006), we repeated the same analysis excluding all trials in which the target appeared in the same quadrant as on the previous trial. Taking repetition priming into account is important because it can lead to distorted results. If repeated responses are particularly fast, then the fact that there are inevitably more such repetitions in the rich than sparse quadrants could artificially increase the probabilistic cuing effect. Figure 2A shows participants' RTs for each quadrant (rich or sparse) and for each epoch after eliminating these trials, and Figure $2 \mathrm{~B}$ shows the difference in RT between the sparse and the rich quadrant (i.e., the magnitude of the probabilistic cuing effect). An ANOVA conducted on the remaining

\footnotetext{
${ }^{1}$ Although the preregistered protocol only considered the analysis of reaction times, to ensure that the results were not driven by a speed-accuracy trade off, we analysed errors in the visual search task is the same way as search times. In the biased stage, the ANOVA yielded a main effect of condition, $F(1,155)=11.11, p=.001, \eta_{\mathrm{p}}^{2}=.07$, indicating higher accuracy when the target appeared in the rich quadrant $(M=.99, S D=$ $0.12)$ than in the sparse quadrant $(M=.98, S D=0.14)$, and a main effect of epoch, $F\left(8.69,1346.95=5.38, p<.001, \eta_{\mathrm{p}}^{2}=.03\right)$, indicating lower accuracy for the first six epochs $(M=.98, S D=0.13)$ than for the last six $(M=.99, S D=0.12)$. All other effects were non-significant ( $p$ 's $>.235$ ).
} 
RTs yielded significant effects of quadrant, $F(1,155)=282.09, p<.001, \eta_{\mathrm{p}}^{2}=.65$, and epoch, $F(7.32,1133.83)=58.87, p<.001, \eta_{\mathrm{p}}^{2}=.28$. No other effects were significant $(p$ $>$.147). Overall, these analyses confirm that the rich quadrant acquired a visual search advantage compared to the sparse quadrants even when repetition priming effects were controlled for, and that this effect did not differ across groups.

Unbiased stage. We conducted a similar ANOVA on RTs from the unbiased testing stage. This analysis aims to test the inflexibility hypothesis: If the probabilistic cuing effect is hard to modify, we should find just a quadrant effect. On the other hand, if participants can flexibly adapt their search strategies to reflect the changed quadrant probabilities in the new unbiased stage (falling from $50 \%$ to $25 \%$ for the previously rich quadrant and rising from $16.7 \%$ to $25 \%$ for the previously sparse quadrants), then we should find a significant epoch $\times$ quadrant interaction, produced by the progressive reduction of the probabilistic cuing effect through the unbiased stage. The ANOVA yielded significant effects of quadrant, $F(1,155)=71.22, p<.001, \eta_{\mathrm{p}}^{2}=.32$, and epoch, $F(7.52,1166.22)=8.99, p<.001, \eta_{\mathrm{p}}^{2}=.06$. No other effects were significant $(p>$ .097). After excluding trials in which the target appeared in the same quadrant as on the previous one, the ANOVA yielded significant effects of quadrant, $F(1,155)=69.70, p<$ $.001, \eta_{\mathrm{p}}^{2}=.31$, and epoch, $F(7.89,1224.19)=7.97, p<.001, \eta_{\mathrm{p}}^{2}=.05$, as well as a significant quadrant $\times$ epoch interaction, $F(9.96,1543.03)=1.96, p=.028, \eta_{\mathrm{p}}^{2}=.01$. No other effects were significant $(p>.311)^{2}$. This result suggests that the advantage of the rich over the sparse quadrants is reduced during the unbiased stage (see Figure 2B),

\footnotetext{
${ }^{2}$ Analyses of error rates for the Unbiased stage yielded no significant effects ( $p$ 's $>$ $.112)$.
} 
although the interaction only reached statistical significance once repeated trials were removed from the sample.
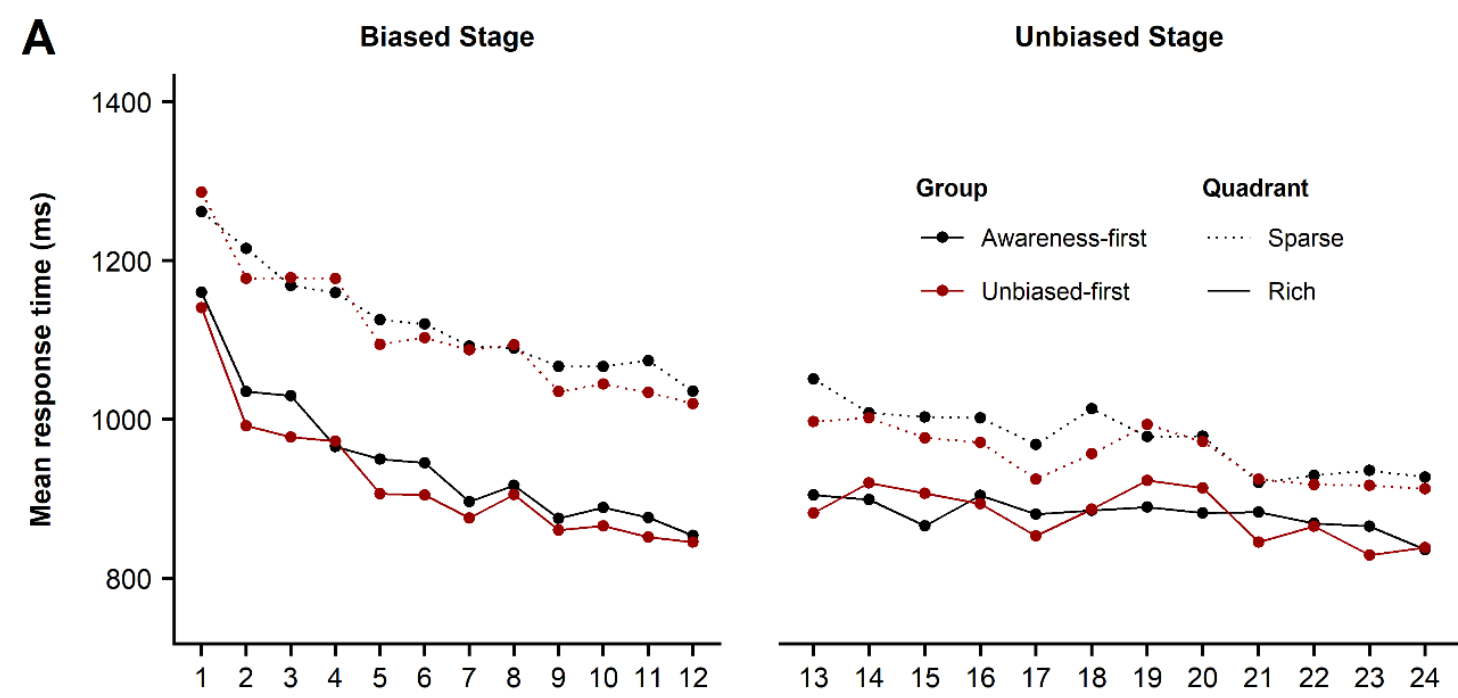

B

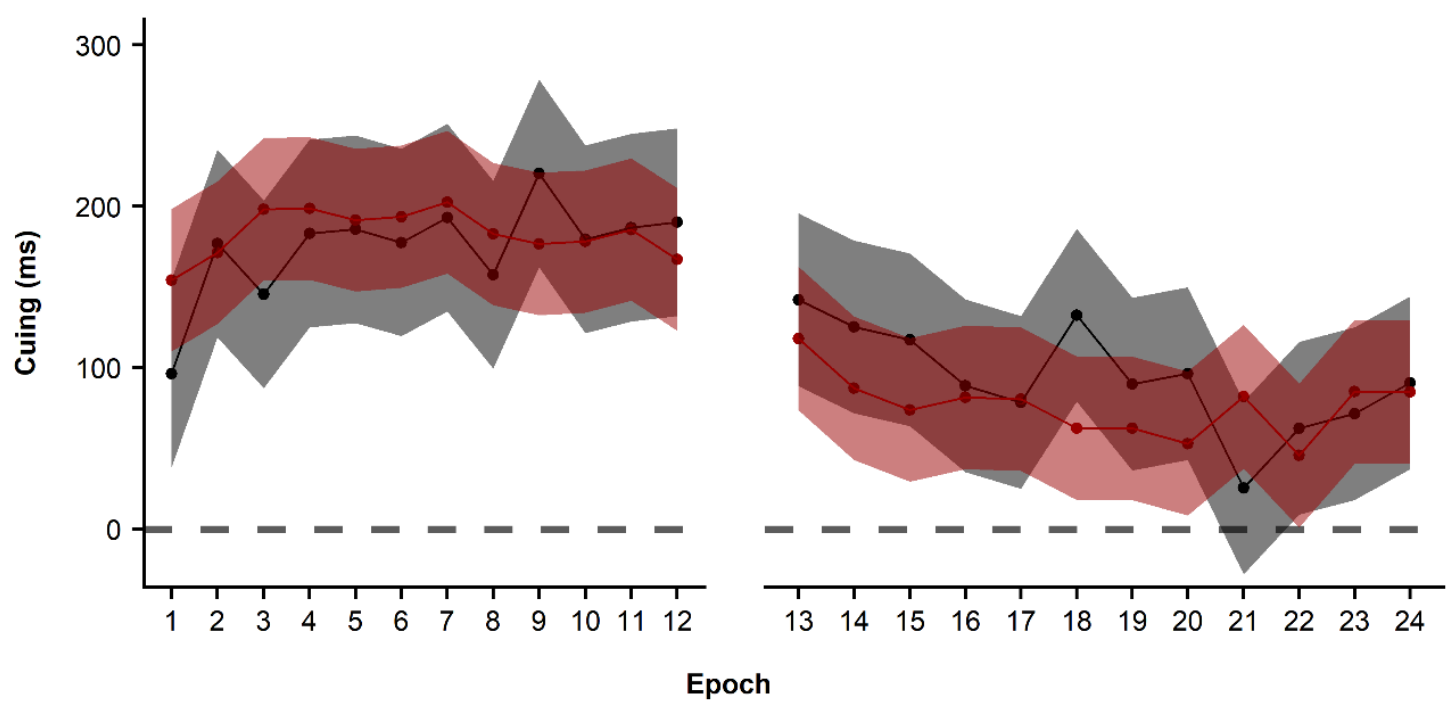

Figure 1. Probabilistic cuing effect after excluding trials in which the target appeared in the same quadrant as on the previous trial. Each epoch comprises two blocks of trials. Panel A: Mean response times for each trial type (target in the rich or sparse quadrant) and group (Awareness-first and Unbiased-first). B: Mean response time difference and 95\% confidence interval between the sparse and rich quadrants (cuing effect) separated by group (Awareness-first and Unbiased-first). The gap in the horizontal axis represents the point at which awareness was tested in the Awareness-first group.

Awareness test. To test whether the presence of an unbiased stage modulated the results of the awareness test, we began by analyzing participants' responses to the 
first question (“Did the T appear with greater probability in any quadrant?"). Their 1-6 numeric responses were submitted to a Mann-Whitney U test, which showed that the Awareness-first group had higher scores than the Unbiased-first group, $Z=2.01, p=$ $.044, r=.16$, although the median was 4 ("Possibly yes") for both groups. Figure 3A shows the proportion of participants in each group who selected each option of the scale.

In the second awareness question, participants ranked the four quadrants in terms of their probability of containing the target. To test which group was more accurate in ranking the quadrants, we coded participants' responses as follows: Participants ranking their rich quadrant as the first, second, third and fourth were assigned a score of 4, 3,2 and 1, respectively, so that higher scores indicate more accurate responses. Although the Awareness-first group had slightly higher scores $(M=$ 3.85) than the Unbiased-first group $(M=3.58)$, a Mann-Whitney $\mathrm{U}$ test found that this difference was not significant, $Z=0.90, p=.367, r=.07$, with a median of 4 (maximum score) for both groups. Figure 3B shows the proportion of participants in each group that obtained a score of 1, 2, 3 and 4 .

To determine whether the proportion of participants ranking the rich quadrant as first, second, third, or fourth was independent of the presence or absence of the unbiased test stage we also carried out a Chi-square test. This test did not yield a significant result, $\chi^{2}(3)=3.73, p=.292, w=0.15$. In order to explore whether the proportion of participants correctly selecting the rich quadrant was above chance, we carried out a two-tailed test of equal proportions for both groups. This analysis was not preregistered. For both the Awareness-first group, $\chi^{2}(1)=67.85, p<.001, w=0.93,95 \%$ Confidence Interval (CI) [54.33, 74.99], and the Unbiased-first group, $\chi^{2}(1)=46.52, p<.001, w=$ 
$0.77,95 \%$ CI $[47.21,68.47]$, the proportion of participants selecting the rich quadrant as the most probable was above chance.

A

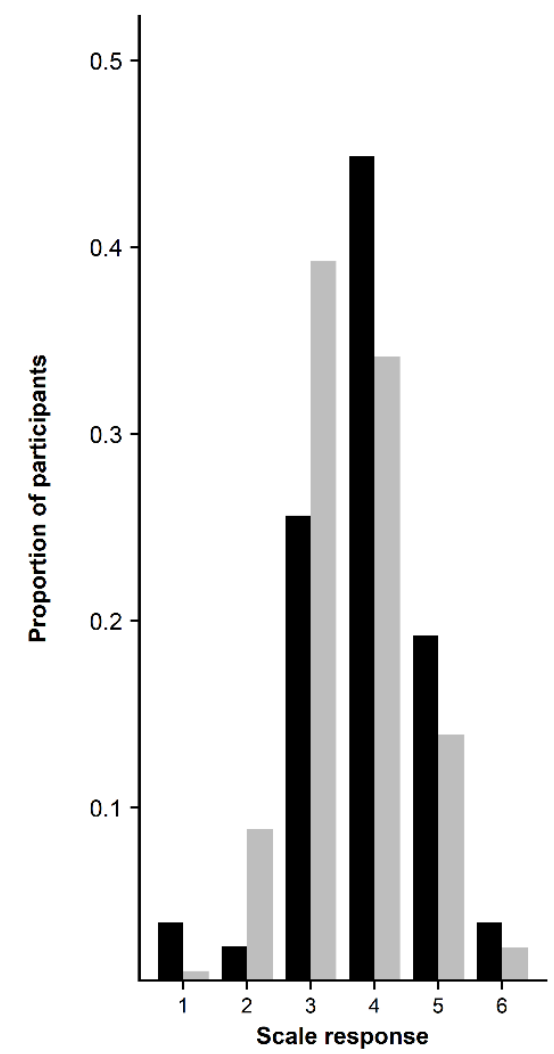

B

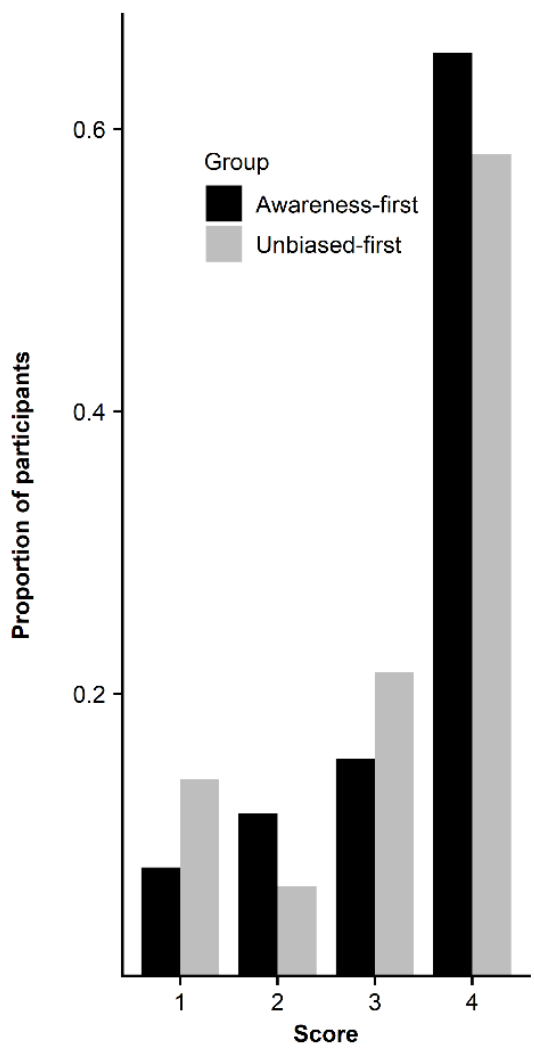

Figure 3. Panel A: Proportion of participants in each group selecting an option from 1 to 6: "definitely not", "probably not", "possibly not", "possibly yes", "probably yes", and "definitely yes" (question 1 of the awareness test). B: Proportion of participants in each group who obtained a score of 1,2, 3 and 4. Scores were assigned considering participants' ranking of quadrants. Those participants ranking their rich quadrant as the first, second, third and fourth most probable quadrant were assigned scores of $4,3,2$, and 1 , respectively.

\section{Experiment 2}

In Experiment 1, analysis of visual search times supported the hypothesis that the bias learnt during the biased stage was attenuated during the unbiased stage, challenging the idea that this bias is driven by an inflexible mechanism. Contrary to the hypothesis that this type of learning is implicit or unconscious, participants in both groups showed above-chance scores for the awareness questions. Importantly, the presence of an unbiased testing stage affected participants' level of awareness. These 
results are the first direct experimental confirmation of the hypotheses put forward by Vadillo, Linssen et al. (2020) in the light of their meta-analytic results.

The absence of a significant result in the second awareness question (i.e., the ranking test) might be seen as a caveat against our hypothesis. However, we suspect that the ranking test (the second awareness question) used in Experiment 1 was not the most appropriate for distinguishing between groups because, from a strictly rational point of view, it required the same response in both groups. By the time a participant in the Awareness-first group reached the awareness test, the target had appeared in the rich quadrant on $50 \%$ of the trials, and by the time a participant in the Unbiased-first group responded to the awareness test, the target had appeared in the rich quadrant on $37.5 \%$ of the trials (the average of $50 \%$ in the biased stage and $25 \%$ in the unbiased stage). Therefore, when asked to rank which quadrant contained the target most often, both groups should provide the same response, ranking the rich quadrant (for the Unbiasedfirst group, meaning the quadrant that was rich in the biased stage) first. This might explain why we failed to detect any difference between the groups on this measure.

In Experiment 2 we attempted to overcome this limitation. This study was an exact replication of Experiment 1 except that the quadrant-ranking test was replaced by a new question asking participants to estimate the relative frequency with which each quadrant had contained the target. The preregistered protocols (methods and analysis plan) are available at https://osf.io/56xpv/.

\section{Method}

\section{Participants}

One hundred and sixty-one participants took part in Experiment 2. As explained in the preregistered protocol, it was not possible to run an informed power analysis for the new second question of the awareness test, as it had never been used in previous 
research. Thus, we calculated statistical power considering (a) the significant quadrant $x$ epoch interaction detected in the unbiased testing stage of Experiment 1, and (b) the first question of the awareness test of Experiment 1. A power analysis revealed that a sample of 160 participants would grant .87 power to replicate the significant quadrant $\times$ epoch interaction detected in the unbiased testing stage of Experiment 1, that yielded a $\eta_{\mathrm{p}}^{2}$ value of .01.

All participants had normal or corrected to normal vision and completed the experimental task individually in isolated cubicles. All participants were Psychology students from Universidad Autónoma de Madrid (UAM), provided informed consent and received course credit for their participation. The study was approved by the UAM Ethics Committee (ref. CEI-80-1473).

\section{Procedure}

The stimuli, apparatus, procedure and design were identical to those of Experiment 1, except for the second question of the awareness test. In Experiment 2, after participants had answered the first question of the awareness test (i.e., "Did the T appear with greater probability in any quadrant?"), we asked them to estimate how often the target had appeared in each quadrant. To this aim, we showed them a square representing the search display divided into four quadrants, together with the following instructions: "Please estimate the percentage of times that the T appeared in each quadrant" ["Por favor, indica el porcentaje de veces que ha aparecido la T en cada uno de los cuadrantes"] (Figure 4). Unlike in Experiment 1, participants were not informed that the location of the target was biased. If the sum of percentages provided by the participant was different from 100, they were asked to revise their estimates. 


\section{Experiment 2}

\begin{tabular}{|c|c|c|c|c|c|c|}
\hline \multirow{2}{*}{ Unbiased-first } & ${ }_{-1}^{50 \%}$ & $r_{\perp} \quad 16 \%$ & $\begin{array}{r}25 \% \\
+ \\
\end{array}$ & r & \multicolumn{2}{|c|}{$\begin{array}{l}\text { Question 1: Did the T } \\
\text { appear with greater } \\
\text { probability in any } \\
\text { quadrant? }(1=\text { definitely } \\
\text { not, } 6=\text { definitely yes). }\end{array}$} \\
\hline & $\begin{array}{ll}16 \% & \\
& \text { ᄀ } \\
& \Gamma\end{array}$ & ـ & $\begin{array}{ll}25 \% & \\
& \neg \\
& \ulcorner\end{array}$ & 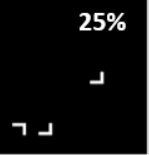 & \multicolumn{2}{|c|}{$\begin{array}{l}\text { Question 2: Please } \\
\text { estimate the percentage of } \\
\text { times that the } T \text { appeared } \\
\text { in each quadrant. }\end{array}$} \\
\hline \multirow{2}{*}{ Awareness-first } & $\begin{array}{r}4 \\
50 \% \\
\end{array}$ & ז & \multirow{2}{*}{\multicolumn{2}{|c|}{$\begin{array}{l}\text { Question 1: Did the T } \\
\text { appear with greater } \\
\text { probability in any } \\
\text { quadrant? ( } 1=\text { definitely } \\
\text { not, } 6=\text { definitely yes). } \\
\text { Question 2: Please estimate } \\
\text { the percentage of times } \\
\text { that the T appeared in each } \\
\text { quadrant. }\end{array}$}} & $\begin{array}{l}25 \% \\
r \\
r\end{array}$ & 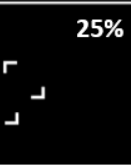 \\
\hline & $\begin{array}{rr}16 \% & \\
& \\
\lrcorner & \Gamma \\
& \\
\end{array}$ & $\begin{array}{ll} & 16 \% \\
& \lrcorner \\
\neg ~ & \\
\end{array}$ & & & 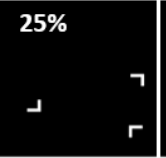 & $\begin{array}{ll} & 25 \% \\
& \lrcorner\end{array}$ \\
\hline
\end{tabular}

Figure 4. Design of Experiment 2. Dividing lines demarcate the four quadrants and percentages indicate the probability of the target being displayed in each quadrant on each trial. Neither the lines nor the percentages were visible to participants. The Unbiased-first group (top row) completed a biased stage followed by an unbiased stage. Then, they took the awareness test. The Awareness-first group (bottom row) took the awareness test between the biased and unbiased stages.

\section{Results and Discussion}

Search times. Participants and search times were filtered as in Experiment 1.

Participants with overall accuracies below $95 \%$ in the visual search task (two in total, one in the Awareness-first group and one in the Unbiased-first group) were removed from the analyses, leaving 78 participants in the Awareness-first group and 81 in the Unbiased-first group.

Biased stage. Probabilistic cuing of attention during the biased stage was assessed by means of a 2 (Group: Awareness-first vs. Unbiased-first) $\times 2$ (Quadrant: Rich vs. Sparse) $\times 12$ (Epoch) ANOVA on RTs. This yielded significant main effects of quadrant, $F(1,157)=419.95, p<.001, \eta_{\mathrm{p}}^{2}=.73$, and epoch, $F(7.82,1227.90)=87.66$, 
$p<.001, \eta_{\mathrm{p}}^{2}=.36$, and a significant epoch $\times$ quadrant interaction, $F(9.77,1533.58)=$ $3.13, p<.001, \eta_{\mathrm{p}}^{2}=.02$. No other effects were significant $(p>.075)$.

As in Experiment 1, we repeated the same analysis excluding all trials in which the target appeared in the same quadrant as on the preceding trial. Figure 5A shows participants' RTs for each quadrant and epoch after removing these trials and Figure 5B shows the difference between RTs for the sparse and the rich quadrant (i.e., the cuing effect). The ANOVA yielded significant effects of quadrant, $F(1,157)=269.47, p<$ $.001, \eta_{\mathrm{p}}^{2}=.63$, and epoch, $F(8.23,1291.80)=76.91, p<.001, \eta_{\mathrm{p}}^{2}=.33$, as well as significant group $\times$ quadrant, $F(1,157)=4.97, p=.027, \eta_{\mathrm{p}}^{2}=.031$, and quadrant $\times$ epoch interactions, $F(9.79,1537.03)=4.11, p<.001, \eta_{p}^{2}=.026$. No other effects were significant $(p>.419){ }^{3}$ As in Experiment 1, these analyses reveal that the rich quadrant acquired a visual search advantage compared to the sparse quadrant that was more pronounced as epochs progressed. The group $\times$ quadrant interaction was unexpected and probably attributable to sampling error, because at this point of the experiment, both groups had been exposed to identical conditions.

Unbiased stage. Similar ANOVAs were conducted on RTs from the unbiased stage. The ANOVA conducted on RTs from all trials yielded significant effects of quadrant, $F(1,157)=71.80, p<.001, \eta_{\mathrm{p}}^{2}=.314$, and epoch, $F(8.78,1378.15)=5.81, p$ $<.001, \eta_{\mathrm{p}}^{2}=.04$, a significant group $\times$ quadrant interaction, $F(1,157)=6.52, p=.012$,

\footnotetext{
${ }^{3}$ Again, we addressed the possibility that the effects observed in RTs are driven by a speed-accuracy trade off. In the Biased stage, this analysis yielded a main effect of quadrant, $F(1,157)=25.38, p<.001, \eta_{\mathrm{p}}^{2}=.14$, indicating that accuracy was higher when the target appeared in the rich $(M=.99, S D=0.10)$ than in the sparse quadrant $(M$ $=.98, S D=0.13)$, and a main effect of epoch, $F\left(7.81,1226.17=7.56, p<.001, \eta_{\mathrm{p}}^{2}=\right.$ .05 , indicating that the task was more difficult in the first $(M=.98, S D=0.12)$ than in the last half $(M=.99, S D=0.11)$. All other effects were non-significant $(p>.109)$.
} 
$\eta_{\mathrm{p}}^{2}=.04$, and a significant quadrant $\times$ epoch interaction, $F(10.04,1576.75)=5.98, p<$ $.001, \eta_{\mathrm{p}}^{2}=.04$. No other effects were significant $(p \mathrm{~s}>.124)$.
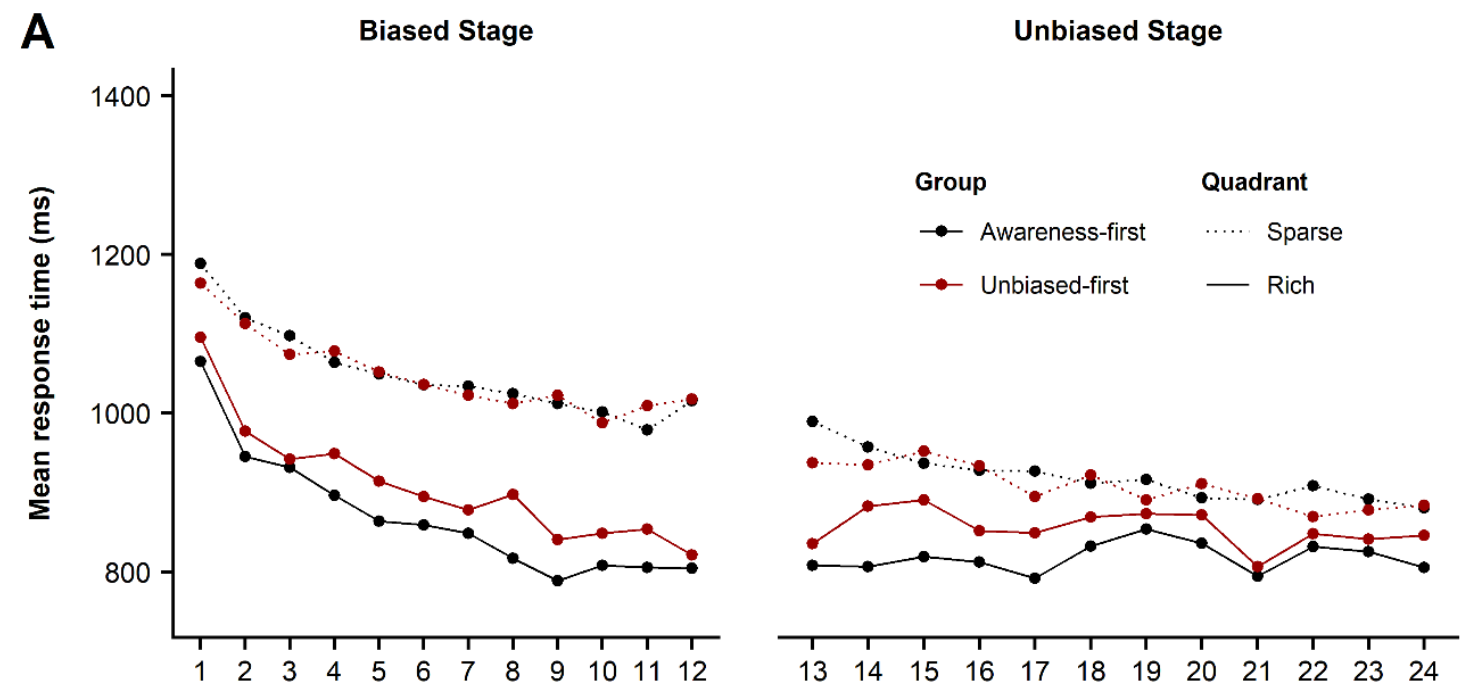

B

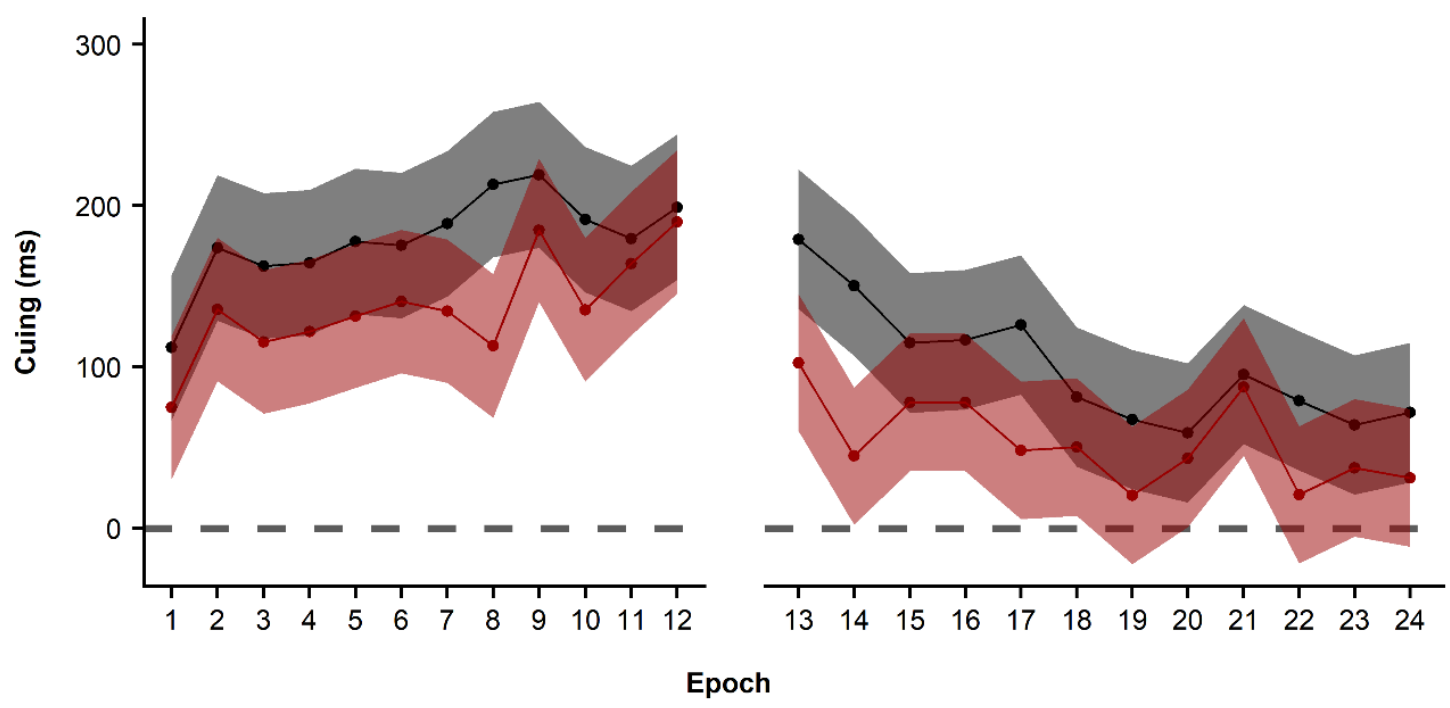

Figure 5. Probabilistic cuing effect after excluding trials in which the target appeared in the same quadrant as on the previous trial. Each epoch comprises two blocks of trials. Panel A: Mean response times for each trial type (target in the rich or sparse quadrant) and group (Awareness-first and Unbiased-first). B: Mean response time difference and 95\% confidence interval between the sparse and rich quadrants (cuing effect) separated by group (Awareness-first and Unbiased-first). The gap in the horizontal axis represents the point at which awareness was tested in the Awareness-first group.

After excluding trials in which the target appeared in the same quadrant as on the previous trial, the ANOVA yielded exactly the same pattern: significant effects of 
quadrant, $F(1,157)=66.59, p<.001, \eta_{\mathrm{p}}^{2}=.30$, and epoch, $F(8.92,1400.60)=4.73, p$ $<.001, \eta_{\mathrm{p}}^{2}=.03$, a significant group $\times$ quadrant interaction, $F(1,157)=6.18, p=.014$, $\eta_{\mathrm{p}}^{2}=.04$, and a significant quadrant $\times$ epoch interaction, $F(11,1727)=5.32, p<.001$, $\eta_{\mathrm{p}}^{2}=.03$. No other effects were significant ${ }^{4}(p \mathrm{~s}>.271)$. As in Experiment 1, these results imply that the search advantage of the rich quadrant over the sparse quadrant is reduced during the unbiased stage (Figure 5). The unexpected effect of group on cuing that we found in the biased stage seemed to persist in the unbiased testing stage, together with a significant group $\times$ quadrant interaction. These effects might simply be due to the unexpected group differences detected in the biased stage. It is also possible that the fact that the Awareness-first group had already taken the awareness test at this stage somehow influenced visual search in subsequent trials. Perhaps participants in this group were biased by the suspicion that the location of the target was unevenly distributed across the search display.

Awareness test. Figure 6A shows the proportion of participants in each group who selected each of the options on the scale, and Figure 6B shows the mean assigned probability for the rich quadrant. As preregistered, we analyzed responses to the first awareness question through a one-tailed Mann-Whitney $U$ test. This analysis found that the Awareness-first group had a higher awareness score ( $M d n=4$, "Possibly yes") than the Unbiased-first group ( $M d n=3$, "Possibly not"), $Z=2.56, p=.005, r=.20$.

For the second awareness question, we compared the Awareness-first and Unbiased-first groups on their percentage estimations for the rich quadrant through a one-tailed independent samples $t$-test. This test revealed that the Awareness-first group made higher estimates for the rich quadrant (34.35\%) compared to the Unbiased-first group $(29.28 \%), t(158)=3.18, p=.002, d=.50$. Together, these results confirm that

\footnotetext{
${ }^{4}$ For the Unbiased stage, no effects involving error rates were significant $(p>.070)$.
} 
participants who completed the awareness test immediately after the unbiased stage showed greater signs of awareness. Of note, $70.51 \%$ of participants in the Awarenessfirst group and $48.15 \%$ of those in the Unbiased-first group assigned greater probability to the rich quadrant than to the sparse ones.

To determine whether participants' estimates were above chance, we carried out non-preregistered two-tailed one sample $t$-tests against the null hypothesis of $25 \%$ for each group. The mean estimate for the rich quadrant made by the Unbiased-first group was higher than chance, $t(80)=4.05, p<.001, d=.45$, as was the estimate made by the Awareness-first group, $t(77)=7.88, p<.001, d=.89$.

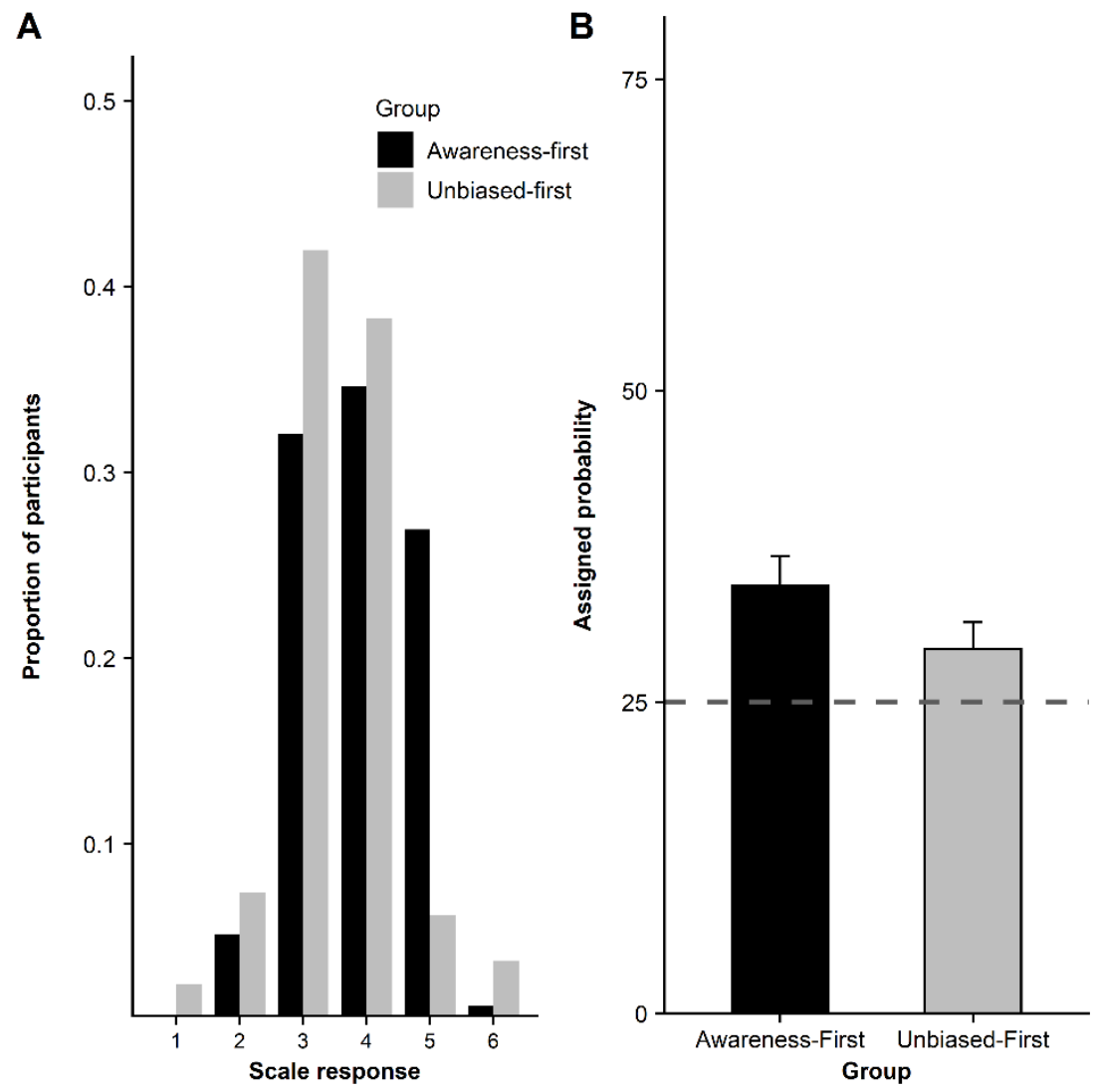

Figure 6. Panel A: Proportion of participants in each group selecting an option from 1 to 6: "definitely not", "probably not", "possibly not", "possibly yes", "probably yes", and "definitely yes" (question 1 of the awareness test). B: Mean (standard error) estimate of the percentage of targets in the rich quadrant. The grey dashed line indicates chance level. 


\section{Non-preregistered analysis}

Evolution of the cuing effect during the task

To further explore the flexibility of probabilistic cuing we carried out several non-preregistered analyses. All of them excluded trials in which the target appeared in the same quadrant on successive trials. First, we wanted to explore the interaction between epoch and quadrant during the unbiased stage. Given that participants in the Awareness first group were aware of the biased distribution of targets before the unbiased stage started and that this could affect visual search (as seemed to be the case in Experiment 2), we eliminated data from this group in the following analyses. To minimize loss of statistical power, we collapsed data from the Unbiased-first groups of both experiments. A 2 (Quadrant: Rich vs. Sparse) $\times 12$ (Epoch) ANOVA on RTs yielded a significant effect of quadrant, $F(1,122)=38.28, p<.001, \eta_{\mathrm{p}}^{2}=.24$, and a significant effect of epoch, $F(11,778.36)=7.25, p<.001, \eta_{\mathrm{p}}^{2}=.06$. Most importantly, the ANOVA also yielded a significant quadrant $\times$ epoch interaction, $F(11,1154.12)=$ $2.58, p=.005, \eta_{\mathrm{p}}^{2}=.02$, suggesting that the difference in RTs between the rich and the sparse quadrant diminished across epochs. Although previous studies have usually found no decrease of cuing over the unbiased stage (e.g., Jiang, Swallow, Rosenbaum et al., 2013), the present results suggest that those studies might simply have been underpowered to detect it.

To explore whether the probabilistic cuing effect was simply reduced or eliminated, we carried out separate paired-samples $t$-tests for each epoch. All the contrasts were significant $(p s<.026)$, suggesting that rich quadrants had an attention advantage over sparse quadrants in all epochs, even at the end of the unbiased stage. This does not mean that the size of this attentional bias was equivalent across all 
epochs. We measured the decrease in the cuing effect by comparing the size of cuing (RTs to targets in the sparse quadrants minus RTs to targets in the rich quadrant) for the first and last two epochs of the unbiased stage. Again, this analysis included participants from the Unbiased-first group of both experiments. It yielded a significant effect, $t(159)$ $=2.12, p=.036, d=.17$, suggesting that the cuing effect was larger during the first two epochs $(M=87.98, S D=162.39)$ than during the last two epochs $(M=59.34, S D=$ 137.22) of the unbiased stage.

It is worth noting that a probabilistic cuing effect was present from the beginning of the task in both experiments (see Figures 2B and 5B). In Experiment 1 a pairedsamples $t$-test on the first epoch of the biased stage collapsing data from both groups revealed a significant difference, $t(156)=6.35, p<.001, d=.51$, in consequence of faster search times for the rich $(M=1146.80, S D=268.28)$ compared to the sparse quadrant $(M=1272.27, S D=266.73)$. In Experiment 2, a similar $t$-test also revealed a significant advantage for the rich quadrant, $t(158)=5.06, p<.001, d=.40$ (rich quadrant: $M=1091.39, S D=270.17$; sparse quadrant: $M=1184.51, S D=249.31$ ). These results suggest that probabilistic cuing developed in the very first 24 trials. In addition, the cuing effect became stronger over the biased stage. A comparison of the cuing effect for the first two epochs versus the last two of the biased stage showed a marginally significant effect in Experiment $1, t(156)=-1.98, p=.052$ (smaller cuing for the first two blocks, $M=149.82, S D=184.85$, than for the last two blocks, $M=182.44$, $S D=172.15)$, and a significant effect in Experiment 2, $t(158)=-3.87, p<.001$ (smaller cuing effect in the first two blocks, $M=123.72, S D=189.29$, than in the last two blocks, $M=183.05, S D=157.51)$.

Relationship between awareness measures and cuing 
We also analyzed the consistency between the different measures of awareness taken in both experiments and their relationship with the size of the probabilistic cuing effect. First, we wanted to know whether those participants who answered with a higher degree of certainty on the scale (question 1 of the awareness test of both experiments) correctly predicted the appropriate quadrant. A Pearson correlation was calculated between scale response and the scores obtained in the ranking test (Experiment 1) and between scale responses and the probability estimations for each quadrant (Experiment 2). We did not find a significant correlation between scale responses and ranking scores in Experiment 1, $r=.09,95 \%$ CI $[-.06, .25], p=.241$, but we did find a significant correlation between scale responses and probability estimates in Experiment 2, $r=.46$, $95 \%$ CI $[.33, .58], p<.001$. The fact that only one of the tests reached significance levels might indicate that the measure of Experiment 2 was more sensitive than the one used in Experiment 1 - because a ranking test just provides an ordinal measure while the probability estimation quantifies the differences between ranking positions.

Secondly, we explored the relationship between performance in the awareness test and probabilistic cuing to elucidate whether explicit knowledge affects responding to targets in the rich quadrant during the visual search task. As a measure of probabilistic cuing, we computed the advantage of the rich quadrant (i.e., response time for the sparse quadrants minus response time for the rich quadrant) over the last six epochs immediately preceding the awareness test (i.e., from the biased stage in the Awareness-first group and the unbiased stage in the Unbiased-first group). In Experiment 1, the Pearson correlation between scores in the ranking test and the magnitude of probabilistic cuing as previously defined was nonsignificant, $r=.04,95 \%$ CI [-.12,.19], $p=.641$. In Experiment 2, in contrast, the correlation between probability estimates for the rich quadrant and the magnitude of probabilistic cuing was significant, 
$r=.23,95 \% \mathrm{CI}[.07-.37], p=.004$. Given that the first question of the awareness test was identical in both experiments, the correlation between cuing and responses to that question can be assessed collating data from both experiments. The correlation between scale responses and probabilistic cuing across participants in both experiments was significant, $r=.13,95 \%$ CI $[.02, .24], p=.018$.

Although not all of these correlations reached statistical significance, overall the analyses reported in this section confirm a considerable degree of association between cuing and awareness.

\section{General Discussion}

Overall, our results challenge the idea that probabilistic cuing is implicit. Previous studies have reported that when participants were asked to answer "yes" or "no" to the question of whether they noticed a bias in the target's spatial distribution, the majority answered "no" (e.g., Jiang, Swallow, Rosenbaum et al., 2013; Jiang, et al., 2014; Jiang, Sha, et al., 2015; Jiang, Swallow, et al., 2015). In contrast, we found that, in general, participants seemed to be aware of the target's unequal spatial distribution. In our Experiment 1, we asked participants to respond to this question using a 5-point confidence scale. The median response for both groups was 4 (“possibly yes”). Furthermore, the studies cited above concluded that when asked to guess the rich quadrant, participants' accuracy is rarely above chance. Although this result applies to many individual studies, the analysis of data from multiple studies usually reaches the opposite result. For instance, after collapsing data from 336 participants, Jiang et al. (2018), found that, in fact, $42.20 \%$ of participants correctly identified the rich quadrant (chance $=25 \%)$. A recent meta-analysis corroborated these results (Vadillo, Linssen et al., 2020). In Experiment 1, we found an even higher recognition rate: Approximately 
$60 \%$ of participants correctly identified the rich quadrant. In Experiment 2, both groups assigned greater frequency of target appearance to the rich quadrant compared to the sparse ones (70.51\% of participants in the Awareness-first group and $48.15 \%$ in the Unbiased-first group). Thus, these findings suggest that previous failures to detect significant levels of awareness in probabilistic cuing experiments are probably attributable to the use of small samples and insensitive dependent measures.

Most of the above-cited experiments included an unbiased stage between the biased stage (where learning about the target's uneven location probability takes place) and the awareness test. This strategy might be diminishing the validity of the awareness assessment because it violates the immediacy criterion (Lovibond \& Shanks, 2002). We found evidence supporting this hypothesis. The measured level of awareness was greater when participants reported their awareness immediately after the biased stage compared to participants who received an unbiased stage before reporting their awareness. Clearly, the unbiased stage - included in many studies with the perfectly reasonable aim of measuring probabilistic cuing uncontaminated by differential target frequencies across quadrants - has a cost: it changes participant's reported awareness. In Experiment 1, the Awareness-first group recorded a higher score on the awareness scale than the Unbiased-first group. In Experiment 2 the Awareness-first group gave a median response of 4 ("possibly yes"), while participants in the Unbiased-first group gave a median response of 3 ("possibly not"). Thus, this manipulation could be the difference between choosing "yes" or "no" in the more traditional test with just two response options. In the same vein, allowing participants to estimate the frequency of appearance of the target in each quadrant revealed that estimates for the rich quadrant were higher in the Awareness-first group than in Unbiased-first group. Altogether, these 
findings motivate our second recommendation, namely that researchers should avoid inserting an unbiased search stage prior to an assessment of awareness.

Finally, using more sensitive measures not only allowed us to detect greater signs of awareness, but also a correlation between awareness and probabilistic cuing. In Experiment 1, responses to the two questions in the awareness test did not correlate with each other; however, they did correlate in Experiment 2. This suggests that the probability ratings collected in Experiment 2 may have provided a more sensitive measure than the scores obtained in the ranking test. Furthermore, we found that, when the immediacy criterion is heeded, scale responses and probability estimates correlate with probabilistic cuing, supporting the hypothesis that this bias is explicit or has an important explicit component. In contrast, responses in the ranking test were not correlated with probabilistic cuing, suggesting that insensitive measures may have contributed to the failure of previous studies to detect a significant correlation between probabilistic cuing and awareness .

Regarding the inflexibility of probabilistic cuing, several experiments that exposed participants to a biased stage followed by an unbiased stage failed to find a significant interaction on RTs between quadrant and epoch in the unbiased stage (Jiang, Swallow, Rosenbaum et al., 2013; Jiang et al.,2014; Jiang, Sha et al., 2015). In contrast, we found a significant interaction demonstrating that the attentional bias acquired in a probabilistic cuing task with one 'rich' quadrant was attenuated during an unbiased target location stage in which the target appeared in each quadrant with equal probability. In our view, the length of the unbiased stage cannot explain the difference in results. Jiang, Swallow, Rosenbaum's et al. (2013) unbiased stage comprised 576 trials and still could not find an interaction between quadrant and epoch. Additionally, some studies have used a shorter unbiased than biased stage (e.g., Jiang, Swallow, \& 
Rosenbaum, 2013; Jiang, Sha et al., 2015), while others have used the same length for both stages (e.g., Jiang, Swallow, Rosenbaum et al., 2013; Jiang et al.,2014; Jiang, Swallow et al., 2015). Thus, this does not seem to explain the difference in results. Instead, we suggest that most of the experiments described in the literature included too few participants to justify any strong inference on the basis of a nonsignificant result. For instance, Jiang et al. (2013, Experiment 1) tested 8 participants; Jiang et al. (2014, Experiment 1) tested 12 participants. In contrast, our sample sizes were roughly 4.5 times larger than those three studies combined, providing sufficient statistical power to detect the crucial interaction between quadrant and epoch in the unbiased stage. Thus, we can make the following straightforward practical recommendation: Researchers should explicitly power their experiments adequately to obtain a significant interaction on RTs between quadrant and epoch in the unbiased stage.

When further exploring this interaction, we found that probabilistic cuing decreased in the last two epochs of the unbiased stage compared to the first two epochs. However, probabilistic cuing was detected in all epochs of the unbiased stage. Thus, the bias is reduced, but did not disappear. Determining how many trials are necessary to fully eliminate the bias is beyond the scope of our study. Future research could usefully address this interesting question.

Our data not only cast doubt on the claim that probabilistic cuing is implicit and persistent, they also challenge the idea that probabilistic cuing is gradual. Cuing increased from the first two epochs of the biased stage to the last two epochs. But in both experiments we found a significant cuing effect from the very first epoch, after only 24 trials. This effect is not new in the literature. For example, Won et al. (2015) found a marginal effect of quadrant even in the first block of 12 trials in a probabilistic 
cuing task undertaken in a natural environment. In the same vein, Salovich et al. (2018) found a search advantage for the high probability quadrant in the first block of 24 trials, even though this block was preceded by 12 unbiased trials. The search advantage was not maintained for the next block, probably due to the presentation of an additional 12 unbiased trials between the first and second blocks. Furthermore, a visual inspection of our data shows that probabilistic cuing declines between the first epoch of the unbiased stage compared to the last epoch of the biased stage. This might suggest that probabilistic cuing is not only rapidly established but also diminishes rapidly. This pattern aligns with the rapid acquisition results noted previously and suggests that even with very limited experience our participants learned the statistical regularities existing in the visual displays and adapted their visuospatial priority map accordingly. This, again, is an argument against the characterization of probabilistic cuing as a gradual and inflexible phenomenon.

It has been proposed that probabilistic cuing reflects habit-like control of selective spatial attention, different from goal-driven and stimulus-driven control mechanisms (e.g., Jiang, 2018; Jiang \& Sisk, 2019; Salovich et al., 2018). This proposal rests on the assumption that it possesses several features of automaticity: that it is gradual, implicit, persistent, insensitive to working-memory load, viewer-centered, and transferable only to other serial search tasks (Jiang \& Sisk, 2019). However, we did not find evidence supporting the claims that probabilistic cuing is persistent and implicit. On the contrary, we found positive evidence of a relationship between explicit knowledge of the spatial distribution of the target and performance in the search task, and we also found evidence of a flexible, and hence possibly controlled, change of spatial attention priority. Therefore, our results suggest that characteristics of 
probabilistic cuing are compatible with a goal-driven mechanism of control of selective spatial attention (Corbetta \& Shulman, 2002).

Interestingly, similar conclusions have been drawn for the contextual cuing effect recently. The contextual cuing effect is another learned visual search bias in which a repeated set of distractors predicts the position of a target. With training, participants learn to locate the target's position in such repeated displays. This search advantage is evident when search time in repeated displays is compared with control trials with new (non-predictive) sets of distractors (Chun \& Jiang, 1998). It has been claimed that contextual cuing, like probabilistic cuing, is implicit and produced partially by "habitual" search responses (Jiang, 2018). Therefore, as in the case of probabilistic cuing, contextual cuing has been characterized not only as implicit but also inflexible (Zellin et al., 2013) and to some extent independent of working memory resources (Vickery et al., 2010) and selective attention (Jiang \& Leung, 2005). However, such claims about the automaticity of contextual cuing have been challenged. Vadillo et al. (2016) showed that participants are aware of the repeated displays when sufficiently sensitive measures of awareness are used in high-powered experiments. Travis et al. (2013) and Vadillo, Giménez-Fernández et al. (2020) found that contextual cuing is not completely independent of working memory or selective attention. Finally, Luque et al. (2017) showed that contextual cuing is flexible and can be modified in a goal-directed way when participants are instructed to search for another target. This is of course an open research topic and the specific contributions of goal-directed and habitual or automatic mechanisms to probabilistic cuing and contextual cuing are still to be fully worked out.

Our results suggest that evidence for implicitness and flexibility is weaker than previously thought. We conjecture that the conflicting results obtained in previous 
studies are due (at least in part) to two methodological shortcomings: lack of adequate statistical power and the inclusion of an unbiased stage prior to the awareness test. Thus, our experiments highlight the advantages of using high-powered preregistered experiments for the study of probabilistic cuing. This might be the path for future probabilistic cuing experiments, especially when null results are theoretically relevant (e.g., awareness tests).

\section{Open Practices Statement}

The data and scripts for both experiments are available at https://osf.io/6ap3f/. 


\section{References}

Chun, M. M., \& Jiang, Y. (1998). Contextual cueing: Implicit learning and memory of visual context guides spatial attention. Cognitive Psychology, 36, 28-71. doi: 10.1006/cogp.1998.0681

Corbetta, M., \& Shulman, G. L. (2002). Control of goal-directed and stimulus driven attention in the brain. Nature Reviews Neuroscience, 3, 201-215. doi:10.1038/nrn755

Geng, J. J., \& Behrmann, M. (2002). Probability cuing of target location facilitates visual search implicitly in normal participants and patients with hemispatial neglect. Psychological Science, 13, 520-525. doi:10.1111/1467-9280.00491

Jiang, Y. V. (2018). Habitual versus goal-driven attention. Cortex, 102, 107-120. doi:10.1016/j.cortex.2017.06.018

Jiang, Y., \& Leung, A. W. (2005). Implicit learning of ignored visual context. Psychonomic Bulletin \& Review, 12, 100-106. doi: 10.3758/s13423-020-01722-x

Jiang, Y. V., Sha, L. Z., \& Remington, R. W. (2015). Modulation of spatial attention by goals, statistical learning, and monetary reward. Attention, Perception, \& Psychophysics, 77, 2189-2206. doi:10.3758/s13414-015-0952-z

Jiang, Y. V., Sha, L. Z., \& Sisk, C. A. (2018). Experience-guided attention: Uniform and implicit. Attention, Perception, \& Psychophysics, 80, 1647-1653. doi:10.3758/s13414-018-1585-9 
Jiang, Y. V., \& Sisk, C. A. (2019). Habit-like attention. Current Opinion in Psychology, 29, 65-70. doi:10.1016/j.copsyc.2018.11.014

Jiang, Y. V., Swallow, K. M., \& Rosenbaum, G. M. (2013). Guidance of spatial attention by incidental learning and endogenous cuing. Journal of Experimental Psychology: Human Perception and Performance, 39, 285. doi:10.1037/a0028022

Jiang, Y. V., Swallow, K. M., Rosenbaum, G. M., \& Herzig, C. (2013). Rapid acquisition but slow extinction of an attentional bias in space. Journal of Experimental Psychology: Human Perception and Performance, 39, 87-99. doi:10.1037/a0027611

Jiang, Y. V., Swallow, K. M., Won, B., Cistera, J. D., \& Rosenbaum, G. M. (2015). Task specificity of attention training: The case of probability cuing. Attention, Perception, \& Psychophysics, 77, 50-66. doi:10.3758/s13414-014-0747-7

Jiang, Y. V., Won, B., \& Swallow, K. M. (2014). First saccadic eye movement reveals persistent attentional guidance by implicit learning. Journal of Experimental Psychology: Human Perception and Performance, 40, 1161-1173. doi:10.1037/a0035961

Lovibond, P. F., \& Shanks, D. R. (2002). The role of awareness in pavlovian conditioning: Empirical evidence and theoretical implications. Journal of Experimental Psychology: Animal Behavior Processes, 28, 3-26. doi:10.1037/0097-7403.28.1.3 
Luque, D., Molinero, S., Watson, P., López, F. J., \& Le Pelley, M. E. (2019). Measuring habit formation through goal-directed response switching. Journal of Experimental Psychology: General. doi:10.1037/xge0000722; 10.1037/xge0000722

Luque, D., Vadillo, M. A., Lopez, F. J., Alonso, R., \& Shanks, D. R. (2017). Testing the controllability of contextual cuing of visual search. Scientific reports, 7:39645. doi: 10.1038/srep39645

Salovich, N. A., Remington, R. W., \& Jiang, Y. V. (2018). Acquisition of habitual visual attention and transfer to related tasks. Psychonomic Bulletin \& Review, 25, 1052-1058. doi:10.3758/s13423-017-1341-5

Travis, S. L., Mattingley, J. B., \& Dux, P. E. (2013). On the role of working memory in spatial contextual cueing. Journal of Experimental Psychology: Learning, Memory, and Cognition, 39, 208-219. doi: 10.1037/a0028644

Vadillo, M. A., Giménez-Fernández, T., Aivar, M. P., \& Cubillas, C. P. (2020). Ignored visual context does not induce latent learning. Psychonomic Bulletin \& Review, 18. doi: $10.1038 /$ srep39645

Vadillo, M. A., Konstantinidis, E., \& Shanks, D. R. (2016). Underpowered samples, false negatives, and unconscious learning. Psychonomic Bulletin \& Review, 23, 87102. doi: $10.3758 / \mathrm{s} 13423-015-0892-6$

Vadillo, M. A., Linssen, D., Orgaz, C., Parsons, S., \& Shanks, D. R. (2020). Unconscious or underpowered? Probabilistic cuing of visual attention. Journal of Experimental Psychology: General, 149, 160-181. doi:10.1037/xge0000632 
Vickery, T. J., Sussman, R. S., \& Jiang, Y. V. (2010). Spatial context learning survives interference from working memory load. Journal of Experimental Psychology: Human Perception and Performance, 36, 1358-1371. doi: 10.1037/a0020558

Walthew, C., \& Gilchrist, I. D. (2006). Target location probability effects in visual search: An effect of sequential dependencies. Journal of Experimental Psychology: Human Perception and Performance, 32, 1294-1301. doi:10.1037/00961523.32.5.1294

Wood, W., \& Rünger, D. (2016). Psychology of habit. Annual Review of Psychology, 67, 289-314. doi:10.1146/annurev-psych-122414-033417

Won, B. Y., Lee, H. J., \& Jiang, Y. V. (2015) Statistical learning modulates the direction of the first head movement in a large-scale search task. Attention, Perception, \& Psychophysics, 77, 2229-2239. doi: 10.3758/s13414-015-0957-7

Zellin, M., Conci, M., von Mühlenen, A., \& Müller, H. J. (2013). Here today, gone tomorrow-adaptation to change in memory-guided visual search. PloS One, 8:59466. doi: 10.1371/journal.pone.0059466

\section{Author note}

MAV and TGF were supported by grant 2016-T1/SOC-1395 from Comunidad de Madrid (Programa de Atracción de Talento Investigador). MAV and DS were supported by a grant from the United Kingdom Economic and Social Research Council. MAV was also supported by grant PSI2017-85159-P from Agencia Estatal de Investigación and Fondo Europeo de Desarrollo Regional. DL was supported by grant 2017-T1/SOC-5147 from Comunidad de Madrid (Programa de Atracción de Talento Investigador) and grant 
PGC2018-094694-B-I00 from Agencia Estatal de Investigación and Fondo Europeo de Desarrollo Regional. Correspondence concerning this article should be addressed to Miguel A. Vadillo, Departamento de Psicología Básica, Facultad de Psicología, Universidad Autónoma de Madrid, 28049 Madrid, Spain. E-mail: miguel.vadillo@uam.es 